\title{
Xiaoxin Chen: funding remains the greatest challenge to research due to constant improvement of technologies
}

Submitted May 14, 2018. Accepted for publication May 15, 2018.

doi: $10.21037 /$ jtd.2018.05.124

View this article at: http://dx.doi.org/10.21037/jtd.2018.05.124

\section{Editor's note}

It is estimated that, in 2018 , over 17,000 new cases of esophageal cancer will be diagnosed in the United States with over 15,000 deaths relating to this deadly cancer. Even though it only makes up about $1 \%$ of all cancers diagnosed in the United States, it is a worldwide issue that many other regions like Iran, India, northern China, and southern Africa are concurrently facing (1). In recent years, targeted therapy has worked its way up to control the growth, division and spread of cancer cells. More and more funding has been allocated to research that seek to manage esophageal squamous cell carcinoma by a diversity of international funding organizations.

Over the years, Prof. Xiaoxin Chen from the North Carolina Central University, Durham has been researching into the molecular mechanisms, prevention and treatment of different kinds of esophageal diseases. It is such an honor for JTD to interview Prof. Chen this time with an aim to not only share his team's knowledge and stories among the peers across the world, but also to bring better understanding and expand the influence of his research.

\section{Expert introduction}

Xiaoxin Chen, MD, $\mathrm{PhD}$, is currently a Professor of Biological and Biomedical Sciences, Cancer Research Program, Julius L. Chambers Biomedical Biotechnology Research Institute, North Carolina Central University, Durham, North Carolina (Figure 1).

After graduating with a bachelor's degree in Medicine at Peking University Health Sciences Center (formerly Beijing Medical University), Beijing, China in 1991, Prof. Chen left for New Jersey in 1994 to complete his PhD and receive further postdoctoral trainings respectively at the Department of Biochemistry and Department of Chemical Biology at Rutgers University.

Prof. Chen led a team of experts to work on treatment of esophageal diseases with focuses on: (I) molecular

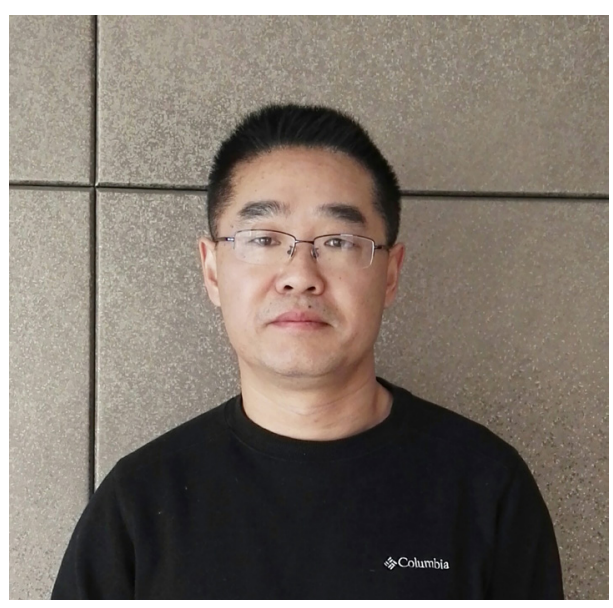

Figure 1 Prof. Xiaoxin Chen.

mechanism, prevention and treatment of gastroesophageal reflux disease, Barrett's esophagus and esophageal adenocarcinoma; (II) preclinical targeted therapy of esophageal squamous cell carcinoma; and (III) molecular mechanism and prevention of alcohol and tobaccoassociated oro-esophageal cancer.

\section{Interview}

FTD: What leads you to the study of gastroesophageal diseases?

Prof. Chen: I spent several years in clinical practice after medical school. I was a resident and research assistant in orthopedic surgery. Joint replacement was the main procedure we used for arthritis patients. So then I wanted to go back to graduate school for a $\mathrm{PhD}$ in immunology. To my disappointment, I was not able to find an interesting immunology lab in my graduate school. Instead a biochemistry professor was looking for a graduate assistant to work on esophageal adenocarcinoma. That project required surgical techniques to create an animal 
model in rats. Because of my background in surgery, I was able to perform a procedure called "esophagoduodenal anastomosis" on more than 30 rats every day. That was a big help for my professor's lab. Finally, I decided to stay in this lab and remain committed to research on gastroesophageal diseases. My mentor, Dr. Chung S. Yang, is a wellestablished leader in esophageal cancer field. Without his mentoring, I would not be able to develop a career in this research field.

\section{FTD: You bave been receiving NIH funding for your research on the management of esophageal cancer. Can you briefly introduce us to your research, e.g. scope, purpose, duration of funding?}

Prof. Chen: My lab has been funded by NIH ever since 2003. We used to focus on mechanism-based chemoprevention of esophageal adenocarcinoma. Over time we moved onto molecular mechanism of Barrett's esophagus and gastroesophageal reflux disease. Now we are mainly focusing on molecular mechanisms and targeted therapy of esophageal squamous cell carcinoma. Our current work is funded by NIH up to 2022. Our first goal is to develop Nrf2 inhibitors for Nrf2-high esophageal squamous cell carcinoma.

\section{FTD: What sort of challenges/difficulties have you encountered during your research?}

Prof. Chen: We have been almost solely replying on NIH funding. Funding is still the greatest challenge. Experimental cost has been rising rapidly due to new technologies. It is great that we can have much better understanding of esophageal cancer with these technologies. On the other hand, high cost is always a prohibitory factor when we design and carry out experiments. It is also a big challenge to find graduate students, postdocs and staff with proper training, experience and dedication.

FTD: What is the current status of this research? And how do you expect it to further develop in the future?

Prof. Chen: Our current research work on esophageal cancer is moving forward smoothly as we would expect. We have generated some exciting and promising data. We would expect translation into clinical studies in the near future.

\section{FTD: In your article "New strategies in esophageal} carcinoma: promises and problems" published in FTD in 2016, your team had examined the problems and promises of targeted therapy for esophageal cancer. Have any of those problems solved so far? (2)

Prof. Chen: We have pointed out several problems in that article published in 2016. Unfortunately, they have not been solved so far. In fact, we cannot expect dramatic improvement in years. Lack of research funding, lack of interest in translational research, and lack of close collaborations between bench scientists and clinicians are all factors contributing to the reality in esophageal cancer field.

\section{FTD: Is targeted therapy still a promising approach to treat esophageal cancer, or has any new approach arisen recently?}

Prof. Chen: Targeted therapy remains a promising and fruitful approach for treatment of esophageal cancer. We have recently developed a preclinical approach to identify Nrf2 inhibitors for Nrf2-high esophageal squamous cell carcinoma. This approach starts from high-throughput screening of chemical compound libraries with an in vitro cell-based assay, and then moves onto in vitro validation. Candidate compounds will be tested in a genetically modified mouse model of Nrf2-high esophageal phenotype. Our goal is to develop a few compounds as Nrf2 inhibitors for esophageal squamous cell carcinoma in the preclinical setting. Other critical target pathways of esophageal squamous cell carcinoma, for example, EGFR/PI3K/Akt/ mTOR pathway, Myc pathway, p53 pathway, hedgehog pathway, Wnt pathway, Notch pathway, and histone modification pathway, can be approached in a similar way. In the end, an arsenal of compounds needs to be developed for clinical studies on esophageal cancer.

FTD: You proposed a strategy for clinical trials on targeted therapy for esophageal cancer using a mechanism of nonresponsiveness to conduct case by case analysis. Would you explicate how this mechanism works?

Prof. Chen: Non-responsiveness is a very complicated issue in clinical treatment of esophageal cancer. We will have to rely on latest technologies, for example, sequencing, in combination with biochemical and pathological analyses, to discover the molecular mechanisms case by case. 
FTD: What would be your advice to your students who would like to become successful researchers in your

field?

Prof. Chen: A young student needs to ask himself or herself a few questions: (I) does my personality fit basic research? A simple personality test can help; (II) am I interested in basic research? Interest will produce dedication in the long run; (III) is there a mentor who can help me develop this career? He or she has to be willing, capable and available. In terms of skills, a young student needs to be reasonably good at critical thinking, critical reading, hands-on benchwork, scientific writing, scientific presentation, and networking. All these skills require years of training by a good mentor.

\section{Acknowledgements}

We would like to express our sincerest gratitude to Prof.
Xiaoxin Chen for sharing his insights and opinions with us.

\section{Footnote}

Conflicts of Interest: The author has no conflicts of interest to declare.

\section{References}

1. American Cancer Society [Internet]. The United States: American Cancer Society; c2018 [cited 2018 May 11]. Available online: https://www.cancer.org/cancer/ esophagus-cancer/about/key-statistics.html

2. Xiong Z, He J, Chen XL. New strategies in esophageal carcinoma: promises and problems. J Thorac Dis 2016;8:E1501-4.

(Science Editor: Brad Li, JTD, jtd@amepc.org)
Cite this article as: $\mathrm{Li} \mathrm{B.} \mathrm{Xiaoxin} \mathrm{Chen:} \mathrm{funding} \mathrm{remains} \mathrm{the}$ greatest challenge to research due to constant improvement of technologies. J Thorac Dis 2018;10(5):E397-E399. doi: 10.21037/ jtd.2018.05.124 